\title{
Stability and Confinement Studies of High-Performance NBI Plasmas in the Large Helical Device Toward a Steady-State Helical Fusion Reactor
}

\author{
Yasuhiko TAKEIRI, Akio KOMORI, Hiroshi YAMADA, Kazuo KAWAHATA, Takashi MUTOH, \\ Nobuyoshi OHYABU, Osamu KANEKO, Sinsaku IMAGAWA, Yoshio NAGAYAMA, \\ Kiyomasa WATANABE, Takashi SHIMOZUMA, Katsumi IDA, Ryuichi SAKAMOTO, \\ Masahiro KOBAYASHI, Akio SAGARA, Kenichi NAGAOKA, Mikiro YOSHINUMA, \\ Satoru SAKAKIBARA, Yasuhiro SUZUKI, Masayuki YOKOYAMA, Shigeru SUDO, \\ Osamu MOTOJIMA, and the LHD Experimental Group \\ National Institute for Fusion Science, 322-6 Oroshi-cho, Toki 509-5292, Japan
}

(Received 25 November 2007 / Accepted 7 March 2008)

\begin{abstract}
Recent progress in plasma performance and the understanding of the related physics in the Large Helical Device is overviewed. The volume-averaged beta value is increased with an increase in the neutral beam injection (NBI) heating power, and it reached $5.0 \%$ of the reactor-relevant value. In high- $\beta$ plasmas, the plasma aspect ratio should be controlled so that the Shafranov shift would be reduced, mainly to suppress transport degradation and the deterioration of the NBI heating efficiency. The operational regime of a high-density plasma with an internal diffusion barrier (IDB) has been extended, and the IDB, which was originally found using the local island divertor, has been realized in the helical divertor configuration. The central density was recorded as high as $1 \times 10^{21} \mathrm{~m}^{-3}$, and the central pressure reached $130 \mathrm{kPa}$. Based on these high-density plasmas with the IDB, a new ignition scenario has been proposed. This should be a scenario specific to the helical fusion reactor, in which the helical ripple transport would be mitigated. A low-energy positive-NBI system was newly installed for an increase in the direct ion heating power. As a result, the ion temperature $\left(T_{\mathrm{i}}\right)$ exceeded $5.2 \mathrm{keV}$ at a density of $1.2 \times 10^{19} \mathrm{~m}^{-3}$ in a hydrogen plasma. Transport analysis shows improvement of ion transport, and the $T_{\mathrm{i}}$-increase tends to be accompanied by a large toroidal rotation velocity of the order of $50 \mathrm{~km} / \mathrm{s}$ in the core region. The plasma properties in the extended operational regime are discussed from the perspective of a steady-state helical fusion reactor.
\end{abstract}

(c) 2008 The Japan Society of Plasma Science and Nuclear Fusion Research

Keywords: Large Helical Device, MHD stability, Shafranov shift, internal diffusion barrier, ion transport, toroidal rotation, impurity hole, helical fusion reactor, neoclassical transport, anomalous transport, core electron-root confinement

DOI: $10.1585 /$ pfr.3.S1001

\section{Introduction}

The Large Helical Device (LHD) is the world's largest superconducting helical device, which started its operation in 1998 [1-3]. The objective of LHD is to demonstrate the high performance of net-current-free heliotron plasmas relevant to a steady-state helical fusion reactor. During nine years of operation, LHD has exploited novel operational regimes related to plasma confinement, MHD stability, and steady-state operation, including plasma-wall interaction $[4,5]$.

Recently, much progress in the plasma performance has been achieved together with the understanding of the physics, mainly due to the upgrade of the heating systems. The volume-averaged $\beta$ value increased with an increase in the neutral beam injection (NBI) heating power, and reached $5 \%$ as a consequence of the enhancement of the negative-NBI power to $14 \mathrm{MW}$. A low-energy positive-

author'se-mail: takeiri@nifs.ac.jp
NBI system has been installed recently for increasing the direct ion heating power, and, as a result, the ion temperature $\left(T_{\mathrm{i}}\right)$ exceeded $5.2 \mathrm{keV}$ at a density of $1.2 \times 10^{19} \mathrm{~m}^{-3}$ in a hydrogen plasma. The distinguishing feature found in LHD is the formation of an internal diffusion barrier (IDB) [6], which is realized by the combination of efficient pumping with the local island divertor (LID) [7] and core fuelling with repetitive pellet injection [8], and it is sustained by high-power NBI heating. In a superdense core (SDC) plasma with the IDB, the central density was recorded as high as $5 \times 10^{20} \mathrm{~m}^{-3}$ [6]. The IDB has also been observed recently in the helical divertor (HD) configuration with a well-conditioned wall. Based on the SDC/IDB plasmas, a new ignition scenario has been proposed, and it should be specific to the helical fusion reactor, in which the helical ripple transport would be mitigated [9]. The inherent advantage for steady-state operation has been demonstrated by the improvement of the 
ion cyclotron range of frequencies (ICRF) and electron cyclotron resonance heating (ECRH) systems. A long-pulse plasma was sustained for $54 \mathrm{~min}$ with ICRF and ECRH, and the input heating energy reached $1.6 \mathrm{GJ}$ [10].

One of the specific characteristics of LHD is the dependence of the plasma properties, such as confinement, transport, MHD stability, and divertor structure, on the magnetic axis position. In the inward-shifted configuration, the transport, including high-energy particle confinement, is improved while the MHD stability is degraded due to the enhancement of the magnetic hill region; this tendency is reversed in the outward-shifted configuration. The most significant achievement in LHD research is the demonstration of the compatibility of reduced transport and MHD stability, which is realized by the sophisticated optimization of the magnetic configuration including the magnetic axis position and the control of the Shafranov shift due to the plasma pressure [11].

In this overview, we present recent developments in the operational regime of the LHD experiments, highlighting mainly the topics of high- $\beta$, high-density with the IDB, and high- $T_{\mathrm{i}}$, and the understanding of the related physics is discussed from the perspective of a steady-state helical fusion reactor.

\section{Large Helical Device and Heating Systems}

The LHD employs a heliotron configuration, and consists of a pair of continuous helical coils, which have an $l=$ $2 / m=10$ poloidal/toroidal field period, and three pairs of poloidal coils [3]. All the coils are superconducting, and provide a wide variety of magnetic configurations through the control of the current ratio of the coils. The magnetic axis position is changed with the poloidal coils, and ranges from 3.5 to $4.1 \mathrm{~m}$ in major radius in the experiments. The averaged minor radius is about $0.6 \mathrm{~m}$ at the maximum and the corresponding plasma volume is about $30 \mathrm{~m}^{3}$, and these are dependent on the magnetic configuration. The magnetic field strength on the axis is $3 \mathrm{~T}$ at the maximum.

The LHD has an intrinsic HD, which is a sort of helically twisted double-null open divertor. In addition, the LHD is equipped with an LID system, which utilizes the $m / n=1 / 1$ magnetic island induced externally with the perturbation coils [7]. The LID facilitates edge plasma control with highly efficient pumping capability.

The main heating system is a negative-ion-based neutral beam injection (negative-NBI) system, which consists of three tangential injectors with a nominal hydrogeninjection energy of $180 \mathrm{keV}[12,13]$. The total injection power achieved is $14 \mathrm{MW}$. A low-energy positive-NBI system, the injection direction of which is perpendicular to the magnetic axis, was operational in 2005 , and $40 \mathrm{keV}-6 \mathrm{MW}$ injection has been achieved [14].

The ECRH system is equipped with 168,84 , and $82.7 \mathrm{GHz}$ gyrotrons, and the total injection power achieved is $2.1 \mathrm{MW}$ [15]. Using antenna systems with quasi-optical mirrors, each microwave is injected as a highly focused Gaussian beam, and the focused location can be changed in the range of $3.5-3.9 \mathrm{~m}$ of the major radius on the equatorial plane.

The ICRF heating system facilitates long-pulse operatio, which is capable of a steady-state injection of $1 \mathrm{MW}$ with four antennas, while the achieved power is $2.9 \mathrm{MW}$ in short-pulse injection [16].

\section{Properties of Reactor-Relevant High- $\beta$ Plasmas}

As described above, the LHD-plasma performance is strongly dependent on the magnetic axis position. Since the heliotron configuration has a weak magnetic shear in the core region and a magnetic hill toward the peripheral region, linear theory indicates that the interchange mode is unstable in the inward-shifted configurations, in which the particle orbit and the transport are better. On the other hand, in the outward-shifted configurations, although the MHD mode is stable due to expansion of the magnetic well region, the particle orbit and the transport are degraded. Through the optimization of the magnetic axis position, it was found that the interchange mode in the core region is stabilized and the Mercier criterion does not prohibit access to higher $\beta$, even in the inward-shifted configuration of the preset vacuum-magnetic axis position of $R_{\mathrm{ax}}^{\mathrm{vac}}=3.6 \mathrm{~m}$ by the spontaneous generation of a magnetic well due to the finite $\beta$ effect [11].

However, as $\beta$ increases further, the Shafranov shift becomes larger. As a result, the transport is degraded and the NBI heat deposition deteriorates due to the enhancement of the orbit loss by the outward shift of the magnetic axis. Moreover, the large Shafranov shift lowers the MHD equilibrium beta limit. Then, the plasma aspect ratio, $A_{\mathrm{p}}$, which can be controlled by changing the current ratio of the three layers of the helical coils, should be optimized [17]. A large $A_{\mathrm{p}}$ reduces the Shafranov shift, but an excessive increase in $A_{\mathrm{p}}$ violates the MHD stability due to suppression of the spontaneous generation of the magnetic well. As a consequence of a series of experiments for $A_{\mathrm{p}}$ optimization including the magnetic field strength, we have achieved $5 \%$ of the volume-averaged beta value, $\langle\beta\rangle$, which is a reactor-relevant value, at $A_{\mathrm{p}}=6.6, B=$ $0.425 \mathrm{~T}$, and $R_{\mathrm{ax}}^{\mathrm{vac}}=3.6 \mathrm{~m}$ with an increased NBI absorbed power of $11 \mathrm{MW}$ [18].

Figure 1 shows discharge waveforms in the high $\beta$ plasma of $\langle\beta\rangle=5.0 \%$ achieved with pellet injection. The tangential NBI power is reduced in the repetitive pellet injection phase for effective penetration of the pellets. At that time, the Shafranov shift is rapidly reduced, and the perpendicular NBI is then injected together with the restoration of the tangential NBI immediately after the end of the pellet injection. Due to the reduction of the Shafranov shift, the perpendicularly injected beam effectively heats 


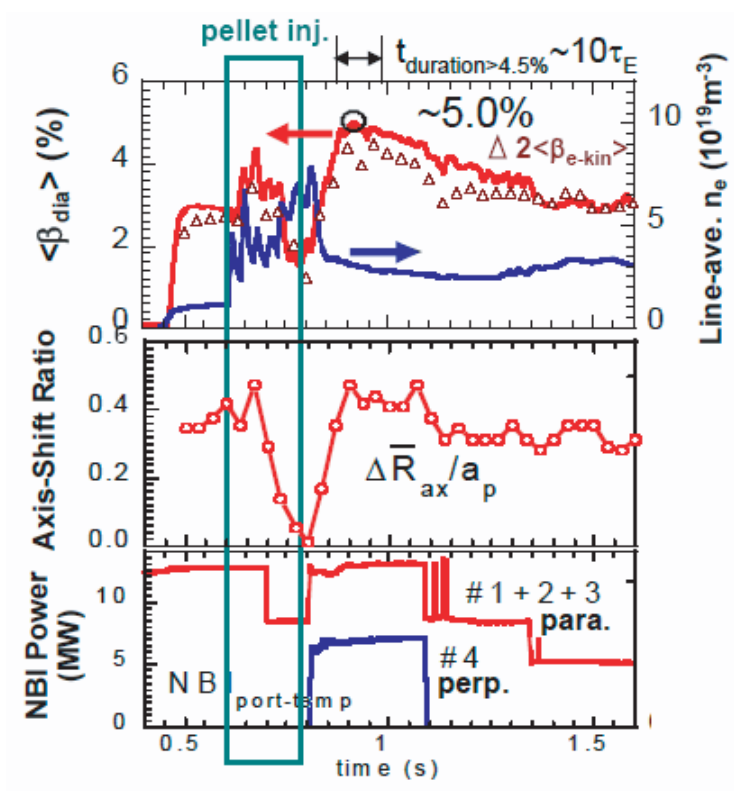

Fig. 1 Waveforms in a high- $\beta$ plasma of $\langle\beta\rangle=5.0 \%$ achieved with repetitive pellet injection. The top plot shows the volume-averaged beta, $\left\langle\beta_{\mathrm{dia}}>\right.$, together with the kinetic value, $\left.2<\beta_{\mathrm{e}-\mathrm{kin}}\right\rangle$, and the line-averaged electron density. The middle plot shows ratio of the magnetic-axis shift to the averaged plasma radius, $\Delta R_{\mathrm{ax}} / a_{\mathrm{p}}$. The bottom plot shows the port-through parallel (tangential) and perpendicular NBI powers. The preset vacuum-magnetic axis position is $3.6 \mathrm{~m}$, plasma aspect ratio is 6.6 , and magnetic field strength is $0.425 \mathrm{~T}$.

the plasma, and $\langle\beta>$ is rapidly increased, finally reaching $5 \%$. Although $<\beta>=5 \%$ was transiently achieved due to the effective fueling by the pellet injection and the effective heating by the perpendicular NBI during the reduction of the Shafranov shift, no crucial MHD instability was observed during a period of 10 times the confinement time, during which the $<\beta>$ was over $4.5 \%$.

With only gas puffing, $<\beta>$ of $4.8 \%$ was stationarily sustained without any disruptive phenomena, as shown in Fig. 2, and over a period of 50 times the confinement time, $<\beta>$ was maintained over $4.5 \%$. The Shafranov shift normalized by the effective plasma minor radius is as large as $\sim 40 \%$, approaching the equilibrium beta limit of $50 \%$. Although the MHD modes excited in the peripheral region with the magnetic hill are dominantly observed, they spontaneously become stable from the inner region to the outer region as $<\beta>$ increases. According to linear MHD analysis, the Mercier criterion is not violated, and the observed peripheral MHD modes in the magnetic hill region are not crucial due to the strong magnetic shear there [19].

Even in the highest- $\beta$ plasma, as described above, the MHD instabilities do not limit the achievable beta, but the heating power does. As shown in Fig. 3, $<\beta>$ increases linearly to 0.25 power of the NBI absorption power without saturation. The absorbed power dependence of $\langle\beta>$ deduced from the energy confinement time scaling ISS95 is $P_{\text {abs }}^{0.4}$, and thus, confinement degradation from the scal-

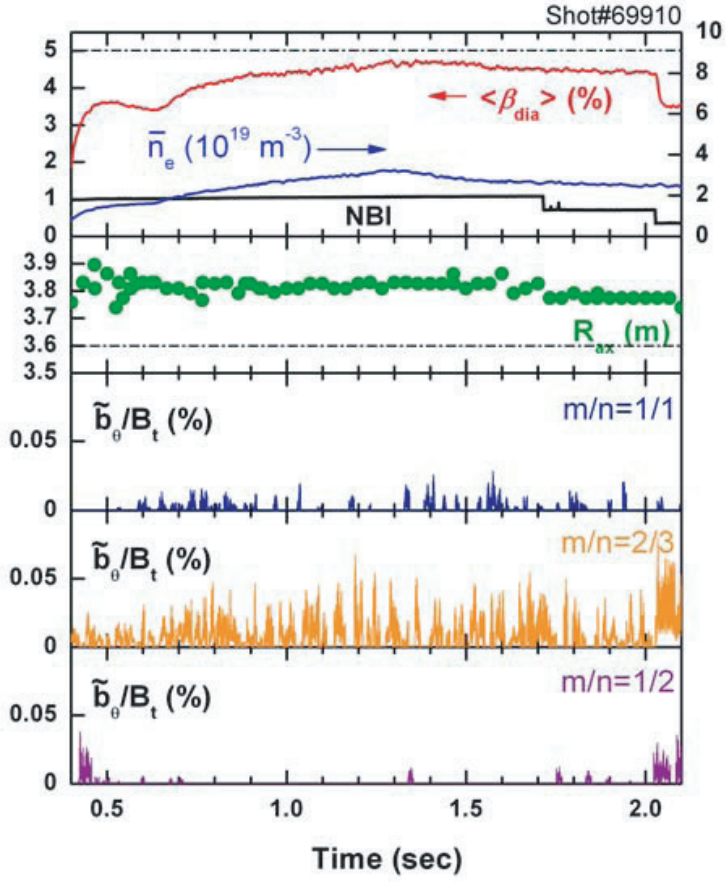

Fig. 2 Waveforms in the stationarily sustained high- $<\beta>$ plasma with only gas puffing. The top shows the volumeaveraged beta, $\left\langle\beta_{\mathrm{dia}}>\right.$, and the line-averaged electron density. The second is the magnetic axis position estimated from the electron temperature and density profiles. The magnetic fluctuations related to the peripheral MHD modes are shown in the three lower figures. The preset vacuum magnetic axis position, $R_{\mathrm{ax}}^{\mathrm{vac}}$, is $3.6 \mathrm{~m}$ and the plasma aspect ratio, $A_{\mathrm{p}}$, is 6.6. The magnetic field strength is $0.425 \mathrm{~T}$ and the NBI absorbed power is $11 \mathrm{MW}$.

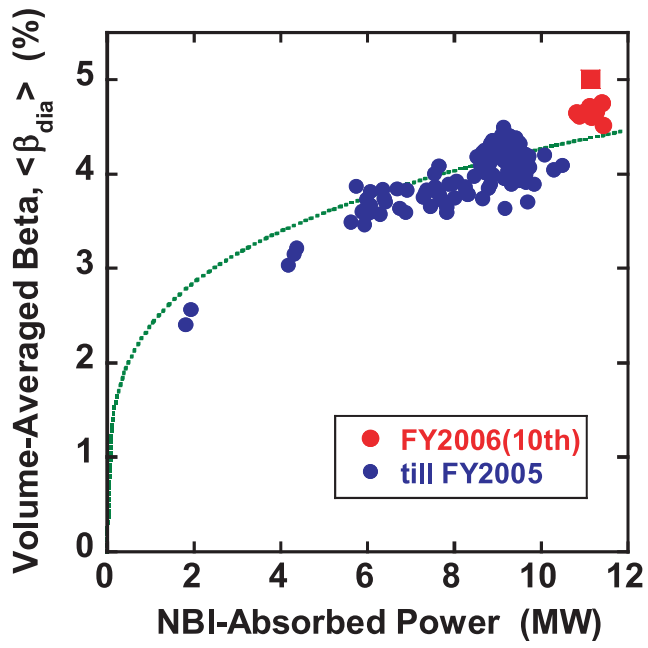

Fig. 3 Volume-averaged beta value, $\left\langle\beta_{\mathrm{dia}}>\right.$, as a function of the NBI-absorbed power. The values obtained in FY2006 are compared with ones obtained up through FY2005.

ing law is suggested in the high- $<\beta>$ plasmas. The peripheral transport is enhanced with an increase in $\langle\beta\rangle$. The dependence on the magnetic Reynolds number, $S$, of the magnetic fluctuation resonating with the peripheral rational surfaces suggests that the observed MHD modes 
are resistive interchange modes [20]. Thus, the resistive g-mode turbulence would cause transport enhancement. Since the $S$ is proportional to $B_{\mathrm{t}} T_{\mathrm{e}}^{3 / 2} n_{\mathrm{e}}^{-1 / 2}\left(B_{\mathrm{t}}\right.$ : magnetic field strength, $T_{\mathrm{e}}$ : electron temperature, and $n_{\mathrm{e}}$ : electron density), the MHD mode amplitude is expected to be reduced for plasmas with higher temperature at higher magnetic field, relevant to reactor-class plasma conditions. Another possible cause for confinement degradation is the increase in the effective helical ripple due to the Shafranov shift [21]. Real-time control of the magnetic axis position during the discharge is planned, which should resolve this issue. It should also be effective in the enhancement of the heating power by the perpendicular NBI, and further increase in $\langle\beta\rangle$ is then expected.

\section{Extension of Operational Regime in High-Density Plasmas with In- ternal Diffusion Barrier}

Formation of the SDC plasma with the IDB is a distinguishing feature in LHD, which never has been observed in tokamaks. The IDB was originally formed in LIDcontrolled plasmas, which are fueled directly in the core region by repetitive pellet injection. The LID functions as a strong pump and realizes a low recycling condition through the particle control at the plasma edge. Even in the HD configuration, intensive wall conditioning should lead to pumping by the wall. In the outward-shifted configuration, the neutrals are not strongly localized in the divertor region, and the recycling is suppressed due to weaker plasma-wall interaction. We have realized IDB formation in the HD configuration in the outward-shifted configuration with such a kind of well-conditioned wall [22].

Figure 4 shows typical waveforms of an SDC plasma with the IDB realized in the HD configuration. The preset vacuum-magnetic axis position, $R_{\mathrm{ax}}^{\mathrm{vac}}$, is $3.8 \mathrm{~m}$. During repetitive pellet injection into the plasma heated with highpower NBI, the electron density is greatly increased, and the central density exceeds $5 \times 10^{20} \mathrm{~m}^{-3}$. After the core fueling with the pellet injection, the peripheral density is rapidly decreased in the density relaxation and temperature recovery phase, and an SDC plasma with the IDB then is formed. The central pressure continues to increase after the plasma stored energy starts to decrease, and occasionally, the core density collapse (CDC) occurs with an abrupt decrease in the central pressure as well as the central density. Impurity accumulation is not observed and $Z_{\text {eff }}$ is as low as around 1.5 during the discharge.

A series of SDC/IDB experiments were performed in the HD configuration by varying $R_{\mathrm{ax}}^{\mathrm{vac}}$, and the results are shown in Fig. 5 [23]. The formation of the IDB is definitely observed in the outward-shifted configuration at $R_{\mathrm{ax}}^{\mathrm{vac}}>$ $3.7 \mathrm{~m}$. The achieved central density, $n_{\mathrm{e}}(0)$, is increased as $R_{\mathrm{ax}}^{\mathrm{vac}}$ is moved outward, and reaches $1 \times 10^{21} \mathrm{~m}^{-3}$ at $R_{\mathrm{ax}}^{\mathrm{vac}}$ $=3.9 \mathrm{~m}$, while the peripheral density remains low, independent of $R_{\mathrm{ax}}^{\mathrm{vac}}$. The central pressure, $P(0)$, jumps up at

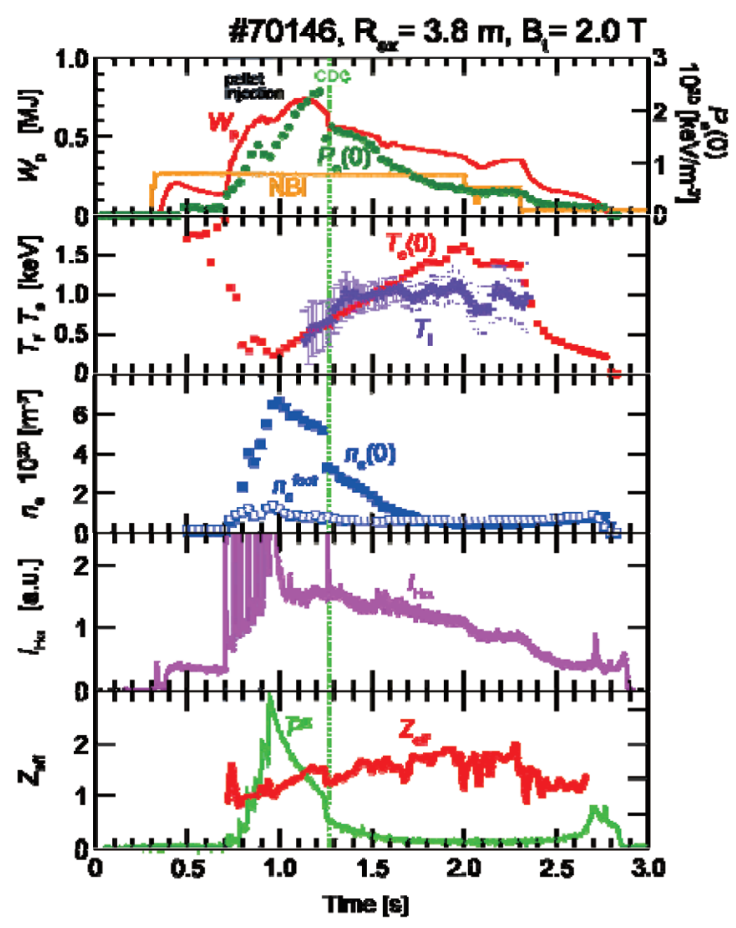

Fig. 4 Typical waveforms of the SDC/IDB plasma formed in the HD configuration. From top: stored energy, $W_{\mathrm{p}}$, and central electron pressure, $P_{\mathrm{e}}(0)$, electron and ion temperatures, central and peripheral (foot) electron densities, $\mathrm{H}_{\alpha}$ intensity, and effective $Z$, and Bremsstrahlung radiation power.

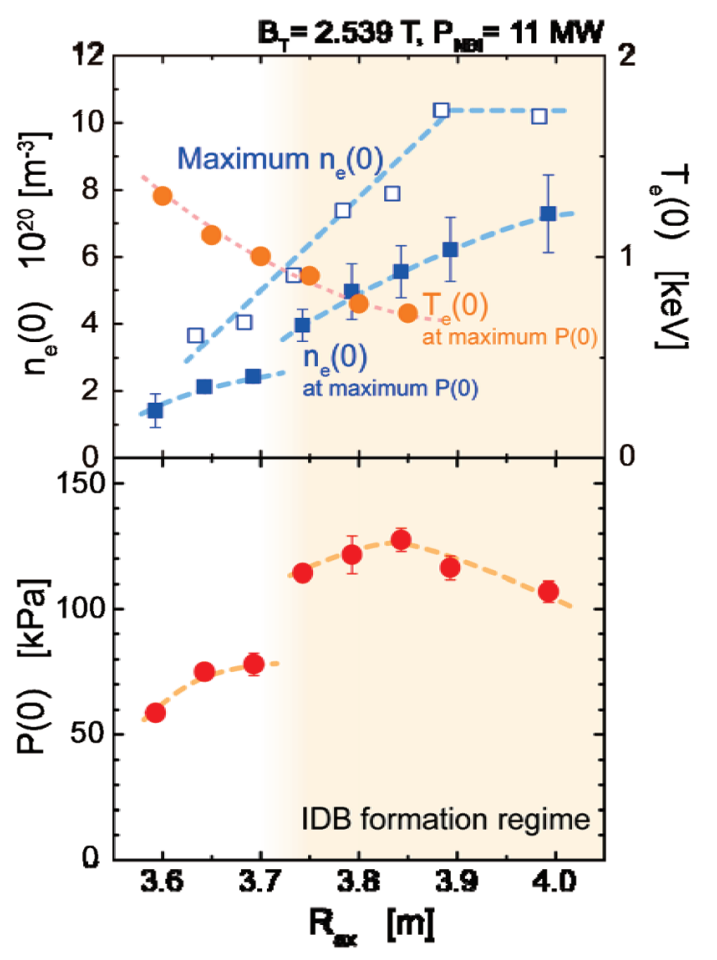

Fig. 5 Maximum central electron density, $n_{\mathrm{e}}(0)$, and maximum plasma pressure, $P(0)$, obtained at various preset vacuum-magnetic axis positions, $R_{\mathrm{ax}}^{\mathrm{vac}}$ [23]. Top: maximum $n_{\mathrm{e}}(0)$ together with $n_{\mathrm{e}}(0)$ and $T_{\mathrm{e}}(0)$ at maximum $P(0)$ as a function of $R_{\mathrm{ax}}^{\mathrm{vac}}$. Bottom: maximum $P(0)$ as a function of $R_{\mathrm{ax}}^{\mathrm{vac}}$. 
the IDB formation, and the maximum $P(0)$ is $130 \mathrm{kPa}$, exceeding atmospheric pressure at $R_{\mathrm{ax}}^{\mathrm{vac}}=3.85 \mathrm{~m}$.

IDB formation is closely correlated with the edgeregion temperature, which is strongly affected by the neutral density there. An increase in the neutral pressure in the SOL region causes an increase in the edge density with enhanced recycling, leading to a decrease in the edge temperature. Then, the heating power is lost by enhanced radiation in the low-temperature region, resulting in radiative collapse [24]. In the outward-shifted configuration, recycling is suppressed more compared with that in the inwardshifted configuration, and thus, the low edge-density can be maintained by the wall-pumping effect without the LID. With a closed HD system equipped with a strong pumping function, the stable IDB discharge should be realized even in the inward-shifted configurations.

Comparison of the pressure profile of the SDC/IDB plasma with that of the usual gas-puffing plasma at $R_{\mathrm{ax}}^{\mathrm{vac}}=$ $3.75 \mathrm{~m}$ is shown in Fig. 6 [22]. The magnetic field strength

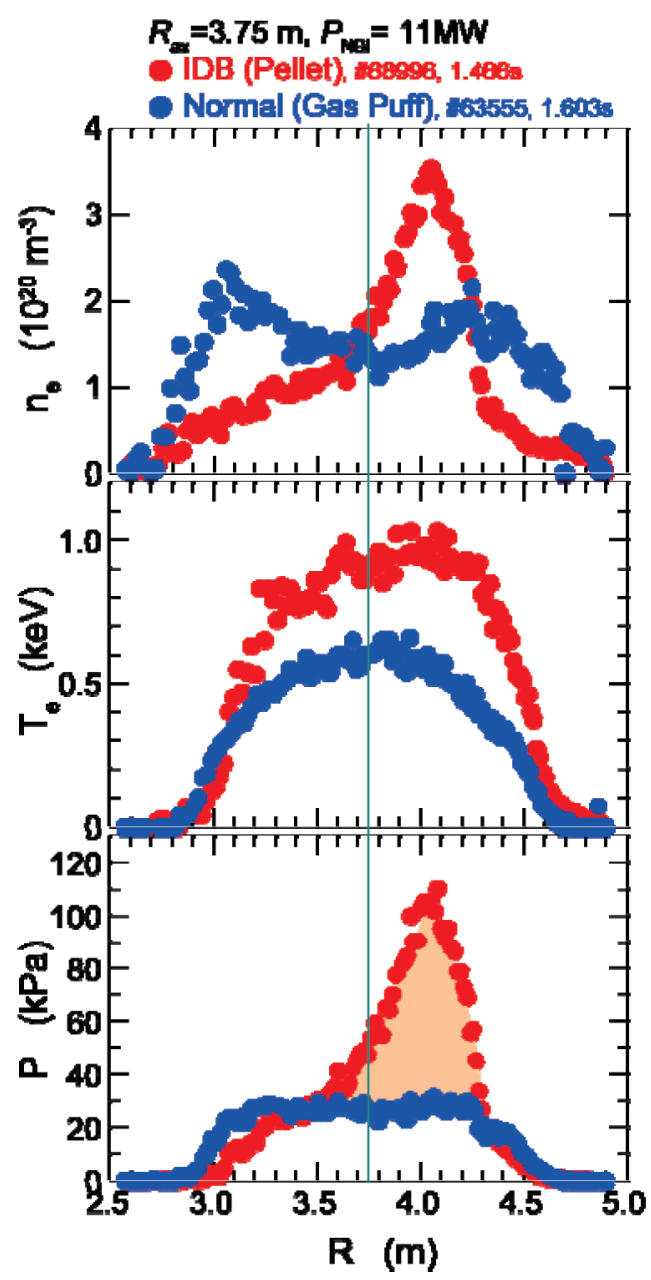

Fig. 6 Comparison of electron density, electron temperature, and plasma pressure profiles of the SDC/IDB plasma with those of the gas-puffing plasma with no IDB [22]. The preset vacuum-magnetic axis position of $R_{\mathrm{ax}}^{\mathrm{vac}}=3.75 \mathrm{~m}$ is indicated by a vertical line. The magnetic field strength and NBI power for both plasmas are the same, $2.539 \mathrm{~T}$ and $11 \mathrm{MW}$, respectively. on the axis and the NBI power are the same for both plasmas, $2.539 \mathrm{~T}$ and $11 \mathrm{MW}$, respectively. Although the central density is about two times higher, the electron temperature is also higher in the SDC/IDB plasma. As a result, the central pressure is much higher with a steep pressure profile, indicating confinement improvement in the SDC/IDB plasma. In the peripheral region, low density is maintained, leading to a high $T_{\mathrm{e}}$-gradient toward the core in the SDC/IDB plasma. On the other hand, since the peripheral density is high and the $T_{\mathrm{e}}$-gradient region is narrow, the core $T_{\mathrm{e}}$ is low in the usual plasma. The central beta value, $\beta(0)$, reaches $4.5 \%$ even in the high magnetic field, and a large Shafranov shift of about $0.3 \mathrm{~m}$ is observed due to the high central pressure with a peaked profile. The achievable $\beta(0)$ is higher at lower magnetic field, and a value of $5.8 \%$ is observed at $B_{\mathrm{t}}=1.5 \mathrm{~T}$. The Shafranov shift is approaching the equilibrium beta limit of $50 \%$ of the effective minor radius. The CDC event shown in Fig. 4 occurs when the Shafranov shift is so large that the shifted axis position would exceed $R=4.0 \mathrm{~m}$. Vertically elongated modification of the magnetic surface by the ellipticity change is applied to suppress the Shafranov shift, and the CDC event is mitigated [25]. Thus, the CDC event is closely related to the Shafranov shift, and the phenomenon of abrupt flushing of the core is thought to be a hard limit near the equilibrium limit. Real-time control of the magnetic axis position by the dynamic control of the vertical field should be effective in improving the SDC/IDB plasma properties.

The achievable plasma stored energy of the SDC/IDB plasmas in outward-shifted configurations is linearly dependent on the plasma volume at the preset vacuum magnetic axis position, $R_{\mathrm{ax}}^{\mathrm{vac}}$, including the non-IDB plasmas in inward-shifted configurations. The plasma volume becomes smaller as the magnetic axis is shifted more outward. Considering that the actual plasma volume is smaller due to the large Shafranov shift in the SDC/IDB plasmas, the plasma confinement is thought to be improved in the SDC/IDB plasmas.

Compared with the high- $\beta$ plasma at low magnetic field, the SDC/IDB plasma has a similar central beta, a steeper pressure gradient, and a larger Shafranov shift. The finite $\beta$ equilibrium in the SDC/IDB plasma at $R_{\mathrm{ax}}^{\mathrm{vac}}$ $=3.85 \mathrm{~m}$ is investigated [26] using the HINT2 equilibrium code in which nested magnetic surfaces are not assumed. The preliminary results show that due to the large Shafranov shift, the magnetic surfaces are distorted in the peripheral region surrounding the IDB, and that inside the IDB, the magnetic surfaces are clearly closed. Since the connection lengths of the magnetic field lines are much longer than the electron mean-free paths even in the region of the distorted magnetic surfaces, it is possible for the electron temperature profile to have a gradient. On the other hand, this phenomenon causes an increase in local transport, and is regarded as a soft limit toward the equilibrium limit, in contrast with the CDC event that is a hard limit. These analyses of MHD equilibria for the SDC/IDB 
plasmas should apply to reactor-relevant high- $\beta$ plasmas.

\section{Increase in Ion Temperature and the Related Ion Transport}

High-energy NBI, which is the main heating device in LHD, dominantly heats electrons, and thus, a high- $Z$ discharge was utilized for high- $T_{\mathrm{i}}$ experiments in LHD to effectively increase the ion heating power. As a result, the $T_{\mathrm{i}}$ was increased with an increase in the ion heating power, and reached $13.5 \mathrm{keV}$ at $0.3 \times 10^{19} \mathrm{~m}^{-3}$ with Ar-gas puffing [27]. To apply this result to the hydrogen discharge, a low-energy NBI system with a radial injection, which dominantly heats ions, has been installed, and $40 \mathrm{keV}$ $6 \mathrm{MW}$ injection was achieved in the last experimental campaign $[14,28]$. This radially injected beam is also utilized for the $T_{\mathrm{i}}$-profile measurement with charge-exchange recombination spectroscopy (CXRS) along a toroidal line of sight, which is better for the measurement in the central region than that along a poloidal line of sight [29].

With a combination of high-energy and lowenergy NBIs, a $5.2 \mathrm{keV}$ ion temperature is obtained at $1.2 \times 10^{19} \mathrm{~m}^{-3}$, as shown in Fig. 7. The density profile tends to be peaked by the low-energy NBI and to be flat or hollow by the high-energy NBI. The peaked density pro-
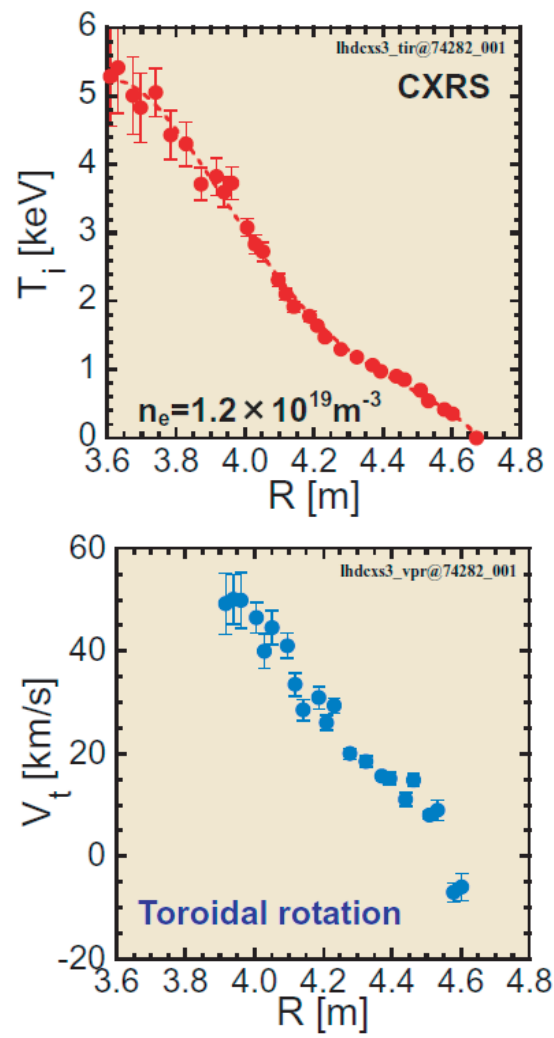

Fig. 7 Profiles of the ion temperature and toroidal rotation velocity in the high- $T_{\mathrm{i}}$ plasma heated with a combination of low-energy and high-energy NBIs. The magnetic axis position is $3.575 \mathrm{~m}$, magnetic field strength is $2.77 \mathrm{~T}$, and electron density is $1.2 \times 10^{19} \mathrm{~m}^{-3}$. file seems to be preferable for $T_{\mathrm{i}}$ rise, suggesting a role of the fueling effect and/or the inward pinch effect in the lowenergy NBI. Figure 7 also shows a profile of the toroidal rotation velocity, $V_{\mathrm{t}}$. The toroidal rotation is enhanced in the same direction as the dominant direction of the tangential NB injectors. As shown in Fig. 7 (b), a large $V_{\mathrm{t}}$ of $50 \mathrm{~km} / \mathrm{s}$ and $V_{\mathrm{t}}$ shear are observed in the core region accompanied by the $T_{\mathrm{i}}$ rise. This suggests a correlation between the ion transport improvement and the toroidal rotation.

Figure 8 shows the results of the transport analysis. The ion thermal diffusivities normalized by $T_{\mathrm{i}}^{3 / 2}$ of the gyro-Bohm factor are shown in Fig. 8 (b) for the plasmas indicated in Fig. 8 (a). It is found that $\chi_{\mathrm{i}} / T_{\mathrm{i}}^{3 / 2}$ is much reduced in $T_{\mathrm{i}}$ rise up to $5 \mathrm{keV}$. As shown in Fig. 8 (c), the neoclassical ambipolar calculation shows negative $E_{\mathrm{r}}$ in the core region in association with the $T_{\mathrm{i}}$ rise, meaning that $T_{\mathrm{i}}$ is increased in the neoclassical ion root. The calculated neoclassical $\chi_{\mathrm{i}}$ is shown in Fig. 8 (d) [30]. Without consideration of the $E_{\mathrm{r}}$ effect, the ripple transport is greatly enhanced by the $T_{\mathrm{i}}$ rise, and it is found that the ripple transport is greatly reduced by the negative $E_{\mathrm{r}}$. As a result, the neoclassical $\chi_{\mathrm{i}}$ is not significantly changed by the $T_{\mathrm{i}}$ rise. Considering that the experimental $\chi_{\mathrm{i}} / T_{\mathrm{i}}^{3 / 2}$ is much reduced, the experimental improvement of the ion confinement is due to the reduction of the anomalous transport in the ion root. The negative $E_{\mathrm{r}}$ is induced by the increased ion temperature, and the role of the negative $E_{\mathrm{r}}$ in the reduction of the anomalous transport should be investigated.

The CXRS intensity profile of the CVI emission shows that the carbon impurity profile becomes strongly hollow as $T_{\mathrm{i}}$ is increased [28]. This "impurity hole" is clearly observed in the plasmas with carbon pellet injection. After carbon pellet injection, $T_{\mathrm{i}}$ increases in the density-decay phase, and the carbon density ratio rapidly decreases to $0.2 \%$ at the plasma center while it is $10 \%$ at the edge. As a result, the $T_{\mathrm{i}}$ measurement in the core region becomes impossible. The outward flux of carbon is observed even with a negative carbon-density gradient. In neoclassical theory, the outward flow of impurities is predicted in the electron root with positive $E_{\mathrm{r}}$, and the impurity pump-out effect is observed in ECRH plasmas. However, the impurity hole is observed in the ion-root plasmas with negative $E_{\mathrm{r}}$. The physics of the impurity hole in high$T_{\mathrm{i}}$ plasmas should be investigated as an inherent subject in helical systems.

On the other hand, $T_{\mathrm{i}}$ rise is also observed in the electron root. When ECRH is superposed on the NBI-heated plasma, $T_{\mathrm{i}}$ is increased accompanied by the formation of the electron-ITB with positive $E_{\mathrm{r}}$ [27]. Transport analysis indicates that both electron and ion transport is improved in the core region with the reduction of anomalous transport. The toroidal rotation is driven in the co-direction by adding ECRH, and it is suggested that the spontaneous toroidal rotation is related to the transport improvement in the electron-root plasma [31]. Although the electron trans- 

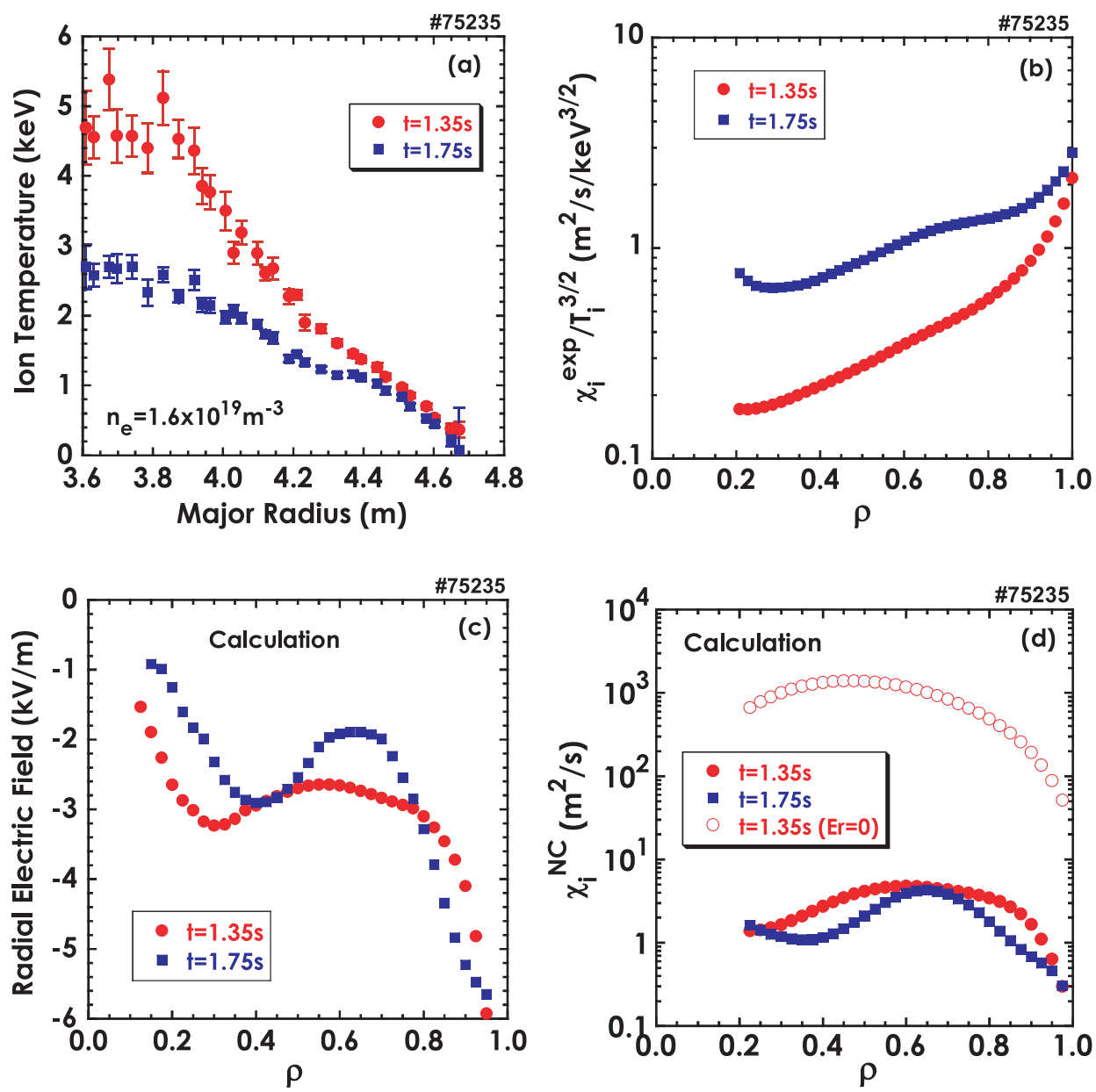

Fig. 8 (a) $T_{\text {i }}$ profiles in the plasmas used for the transport analysis, and (b) the obtained experimental ion thermal diffusivities divided by the gyro-Bohm factor, $\chi_{\mathrm{i}} / T_{\mathrm{i}}^{3 / 2}$. (c) Radial electric field, $E_{\mathrm{r}}$, and (d) the neoclassical ion thermal diffusivities, $\chi_{\mathrm{i}}$, estimated with the neoclassical ambipolar calculation for the plasmas in (a). In (d), $\chi_{\mathrm{i}}$ without consideration of the $E_{\mathrm{r}}$ effect is also indicated.

port improvement is a common feature in the core electronroot confinement (CERC) plasmas [32], the ion transport improvement in CERC suggests a possible approach to achieve reactor-relevant high-temperature plasmas.

\section{High-Density Ignition Scenario for a Helical Fusion Reactor}

For a steady-state helical fusion reactor, a novel ignition approach based on the high-density SDC/IDB plasma is proposed. Figure 9 shows the operational regime for self-ignition based on the high-density IDB profile in a helical reactor. In tokamaks, since high-density operation is limited by current drive condition and MHD stability, a high-temperature path at relatively low density is the most probable ignition scenario. On the other hand, LHD is capable of achieving high-density plasmas above $5 \times 10^{20} \mathrm{~m}^{-3}$ without any limitation, and based on this high-density plasma, the required temperature for the ignition, then, should be reduced below $10 \mathrm{keV}$. This scenario is advantageous for helical devices, which require no current drive. The operation at relatively high collisionality mitigates the helical ripple transport, and the engineering

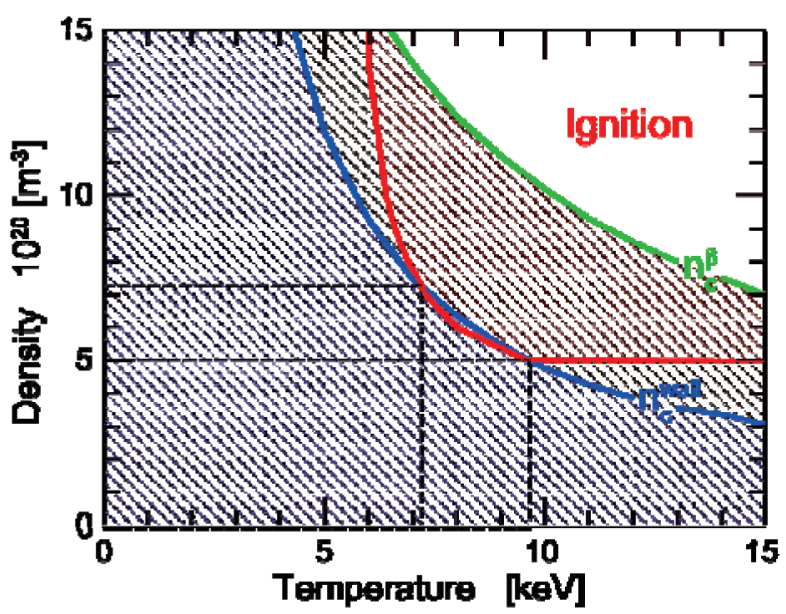

Fig. 9 Operational regime of central density and temperature for high-density ignition in the helical reactor based on the IDB profile. The lines of $n_{\mathrm{c}}^{\text {wall }}$ and $n_{\mathrm{c}}^{\beta}$ indicate critical boundaries for a neutron wall loading less than $2 \mathrm{MW} / \mathrm{m}^{2}$ and a central beta less than $7 \%$, respectively.

demand derived from the high-temperature operation is reduced. In the peripheral region of the SDC/IDB plasma, a 
temperature gradient is established due to the low density there, and a core temperature high enough to realize ignition is achieved by this temperature gradient. Maintaining the low density in the peripheral region is also desirable for avoiding radiative collapse as well as suppression of the enhanced synchrotron radiation. At present, an LHD-type fusion reactor is being designed based on this high-density ignition scenario [9].

\section{Concluding Remarks}

Recent extension of the operational regime in LHD experiments is reviewed together with the progress in understanding the physics. By sophisticated optimization of the magnetic field configuration with regard to the magnetic axis position and plasma aspect ratio, the volumeaveraged beta was increased to $5.0 \%$ with an increase in the NBI heating power. Even in this fusion-relevant high$\beta$ plasma, no crucial MHD instability is observed in the core region while the resistive interchange modes degrade the plasma confinement in the peripheral region. However, these MHD modes should be stabilized in reactor-relevant plasmas with higher temperature at higher magnetic fields.

The SDC/IDB plasmas, which were originally observed in the LID configuration, were realized in the HD configuration. The central density is increased as the preset vacuum-magnetic axis position is moved outward, and reaches $1 \times 10^{21} \mathrm{~m}^{-3}$ at $R_{\mathrm{ax}}^{\mathrm{vac}}=3.9 \mathrm{~m}$. The central pressure exceeds atmospheric pressure and reaches $130 \mathrm{kPa}$. The core plasma confinement is improved while the particle transport in the peripheral plasma surrounding the IDB is degraded. This, in turn, realizes the low density leading to a steep $T_{\mathrm{e}}$-gradient in the peripheral region. The Shafranov shift due to the high central beta and the steep pressure gradient is approaching the equilibrium limit, which probably causes the core density collapse occasionally as a hard limit and the degradation in the outer magnetic surfaces, leading to an increase in local transport, as a soft limit. The dynamic control of the magnetic axis position by the vertical field is also required in high- $\beta$ plasmas.

For an increase in the ion heating power, a low-energy NB injector was newly installed, and $40 \mathrm{keV}-6 \mathrm{MW}$ injection was achieved. The ion temperature was raised to $5.2 \mathrm{keV}$ at $1.2 \times 10^{19} \mathrm{~m}^{-3}$ with the combined heating of the low-energy and high-energy NBIs. Ion transport is improved with the reduction of the anomalous transport in the neoclassical ion root. An increase in the toroidal rotation is observed to be related to the $T_{\mathrm{i}}$ rise. An impurity hole is recognized, in which the carbon impurity is drastically decreased in the core region with an increase in $T_{\mathrm{i}}$. The impurity pump-out effect in the ion root is attractive for the helical reactor because no impurity accumulation is expected in the high- $T_{\mathrm{i}}$ core plasma.

The SDC/IDB high-density plasma allows us to propose a high-density ignition scenario. In this ignition approach, the temperature requirement is reduced below
$10 \mathrm{keV}$ at a high- density above $5 \times 10^{20} \mathrm{~m}^{-3}$, and the helical ripple transport is mitigated together with reduction of the engineering demand caused by the high-temperature operation. Including this scenario, most of the LHD results presented here are relevant to the steady-state helical fusion reactor, and the upgrade of LHD is now planned including deuterium experiments for investigation of higherperformance plasmas, which should lead to a definite design of an LHD-type fusion reactor.

\section{Acknowledgments}

The authors acknowledge all contributions from overseas and domestic collaborators for the progress of the LHD achievements. They are also grateful to the technical staff in LHD for the excellent operation of the LHD and the heating systems.

[1] A. Iiyoshi et al., Fusion Technol. 17, 169 (1990).

[2] A. Iiyoshi et al., Nucl. Fuion 39, 1245 (1999).

[3] O. Motojima et al., Nucl. Fuion 40, 599 (2000).

[4] A. Komori et al., Fusion Sci. Technol. 50, 136 (2006).

[5] O. Motojima et al., Nucl. Fusion 47, S668 (2007).

[6] N. Ohyabu et al., Phys. Rev. Lett. 97, 055002 (2006).

[7] A. Komori et al., Nucl. Fusion 45, 837 (2005).

[8] R. Sakamoto et al., Nucl. Fusion 46, 884 (2006).

[9] A. Sagara et al., Proc. 17th International Toki Conference, Toki, Japan, 2007, I-22.

[10] T. Mutoh et al., Nucl. Fusion 47, 1250 (2007).

[11] H. Yamada et al., Plasma Phys. Control. Fusion 43, A55 (2001).

[12] O. Kaneko et al., Proc. 16th Int. Conf. on Fusion Energy 1996 (Montreal, 1996) Vol. 3 (Vienna, IAEA) p. 539.

[13] Y. Takeiri et al., Nucl. Fusion 46, S199 (2006).

[14] M. Osakabe et al., Proc. 17th International Toki Conference, Toki, Japan, 2007, P2-079.

[15] S. Kubo et al., Plasma Phys. Control. Fusion 47, A81 (2005).

[16] T. Mutoh et al., Nucl. Fusion 43, 738 (2003).

[17] K.Y. Watanabe et al., Nucl. Fusion 45, 1247 (2005).

[18] K.Y. Watanabe et al., Proc. 17th International Toki Conference, Toki, Japan, 2007, I-13.

[19] S. Sakakibara et al., Proc. 21st IAEA Conf. on Fusion Energy (Chengdu, 2006), IAEA-CN-149, EX/7-5 (2006).

[20] S. Sakakibara et al., Fusion Sci. Technol. 50, 177 (2006).

[21] H. Yamada et al., Nucl. Fusion 45, 1684 (2005).

[22] H. Yamada et al., Plasma Phys. Control. Fusion 49, B487 (2007).

[23] R. Sakamoto et al., Plasma Fusion Res. 2, 047 (2007).

[24] J. Miyazawa et al., Plasma Phys. Control. Fusion 48, 325 (2006).

[25] J. Miyazawa et al., Plasma Fusion Res. 3, S1047 (2008).

[26] Y. Suzuki et al., Proc. 17th International Toki Conference, Toki, Japan, 2007, P2-043.

[27] Y. Takeiri et al., Nucl. Fusion 47, 1078 (2007).

[28] K. Nagaoka et al., Plasma Fusion Res. 3, S1013 (2008).

[29] M. Yoshinuma et al., Plasma Fusion Res. 3, S1014 (2008).

[30] S. Matsuoka et al., Plasma Fusion Res. 3, S1056 (2008).

[31] Y. Takeiri et al., Plasma Fusion Res. 3, S1031 (2008).

[32] M. Yokoyama et al., Fusion Sci. Technol. 50, 327 (2006). 